\title{
Acute Spontaneous Subdural Hematoma of Arterial Origin
}

\author{
Joon-Ho Yoon', Young-Bo Shim, Ho-Shin Gwak², Ji-Woong Kwon ${ }^{3}$, Sang-Hyun Lee ${ }^{4}$ \\ ${ }^{l}$ Department of Neurosurgery, Seoul National University College of Medicine, Seoul, \\ ${ }^{2}$ Department of System Cancer Science, Graduate School of Cancer Science and Policy, Goyang, \\ ${ }^{3}$ Department of Neuro-Oncology Clinic, Research Institute and Hospital, Goyang, \\ ${ }^{4}$ Department of Radiology, Research Institute and Hospital, National Cancer Center, Goyang, Korea
}

\begin{abstract}
A spontaneous subdural hematoma (SDH) occurred in a 43-year-old woman during hospital administration. She had received endoscopic thyroidectomy for alleged papillary thyroid carcinoma. By chance, she had a wound infection complicated from esophageal perforation. Therefore, she had to have parenteral nutrition to avoid per os administration of food materials. Five days after the operation, she received central line insertion uneventfully. However, 30 min later, she complained headache and lost consciousness in a few min. Brain CT revealed acute SDH of right hemisphere with severe midline shift. Emergency craniotomy revealed acute SDH. At the end of the removal of hematoma, small cortical artery at temporoparietal lobe was found to be ruptured. Spontaneous SDH form of arterial rupture has been rarely reported. All reported cases have a sudden onset headache followed by neurological deficit mostly progressed to comatose status. The verified origin of bleeding is a cortical artery near the temporal or parietal lobe.
\end{abstract}

Key Words: Hematoma, subdural, acute $\cdot$ Arteries $\cdot$ Hemorrhage

\section{INTRODUCTION}

Acute subdural hematoma (SDH) is commonly caused by traumatic event that tears the bridging veins in the subdural space $^{6}$. Otherwise, some spontaneous SDH cases have been reported to be related with grossly anatomical abnormality such as aneurysm and arteriovenous malformation (AVM). Some other cases are associated medical conditions such as coagulopathy, alcohol overuses, and so on ${ }^{1,9)}$.

Spontaneous acute SDH from arterial bleeding has been rarely reported. Munro first described a patient who had acute SDH without a history of head trauma in 1934. Talalla and McKissock introduced eight cases of acute spontaneous SDH based on autopsy finding of spontaneous rupture of cortical artery. After using computed tomography (CT), many authors have reported cases of spontaneous SDH with cortical artery origin that are confirmed by operative finding ${ }^{1,79)}$. Some cases

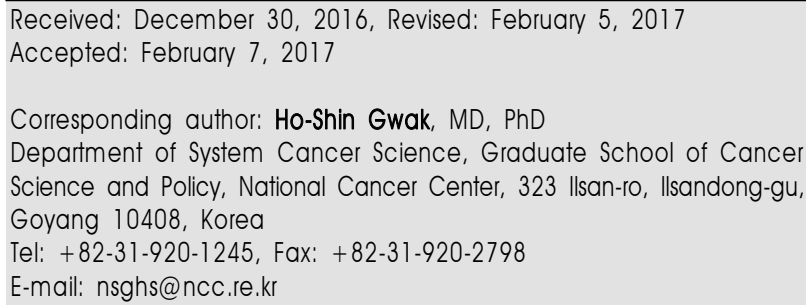

have revealed a leakage of cortical artery via cerebral angiography ${ }^{4)}$.

Here, we report a rare case of spontaneous acute SDH of cortical artery origin confirmed by operative finding.

\section{CASE REPORT}

A 43-year-old woman was hospitalized for surgical removal of thyroid cancer. Her preoperative evaluation for general anesthesia and operation revealed no abnormal finding including coagulopathy. Endoscopic right lobectomy was planned and performed without any noticeable problem. However, on postoperative day one, dirty fluid with foam was noticed through Jackson-Pratt drain. Her amylase level was 32,736 IU/L. Under the impression of esophageal fistulae, nothing per os and total parenteral nutrition (TPN) were prescribed. However, TPN was not admitted due to failure of central line as she could not hold the lying down position due to dyspnea, cough, and sputum. Five days after the operation, central catheter insertion was re-tried and successfully inserted to left subclavian vein on a sitting position.

After returning to the general ward (about 20 min after the procedure), she complained of headache. Her mental status changed to drowsy in five min. Fifty min after the central catheter insertion, patient's mentality became comatose with right 
pupil dilated to $6 \mathrm{~mm}$. She was sent to CT room immediately with oxygen supply via ambu bagging. Her respiratory arrest occurred at CT room. Cardiopulmonary resuscitation was performed for $3 \mathrm{~min}$ which achieved spontaneous respiration. Brain CT revealed acute SDH on right frontotemporoparietal convexity (Fig. 1A).

The patient was immediately transferred to surgical intensive care unit and intubated. Mannitol and steroid were administered. When presented to neurosurgeon, neurological examination revealed fixed pupil of $6 \mathrm{~mm} / 4 \mathrm{~mm}$ and Glasgow Coma Scale (GCS) score of E1VeM2. The patient showed decerebrated posture on pain. Brain magnetic resonance imaging (MRI) including diffusion weighted image was taken to rule out possible underlying parenchymal pathology and to estimate the damage from the herniation. In T2-weighted MRI, the hemorrhage appeared to be isointense mixed with hyperintensity, indicating that the hematoma was a hyperacute stage (Fig. 1B). Fluid-attenuated inversion recovery image showed damaged frontal lobe from subfalcial herniation (Fig. 1C).

Emergency operation was performed about $4 \mathrm{hr}$ after the patient felt the headache. After craniotomy, acute hematoma
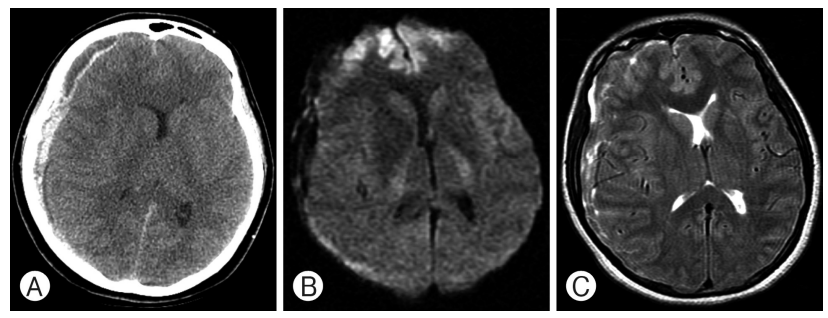

Fig. 1. (A) Acute subdural hematoma at left frontotemporoparietal convexity on computed tomography. (B) High signal intensity of bilateral frontoparietotemporal lobe and right anterior frontal lobe on diffusion weighted image. (C) Low to dark signal intensity at right frontoparietotemporal cerebral convexity on T2-weighted image.
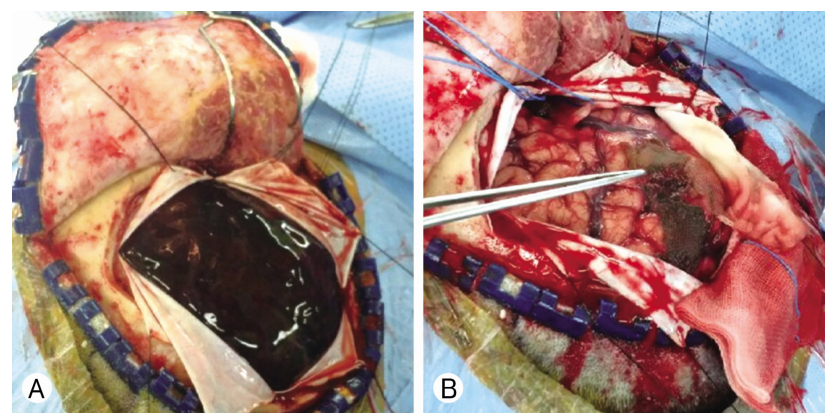

Fig. 2. Operative findings after craniotomy. (A) A large acute hematoma covered the whole right cerebral hemisphere. (B) Cortical arterial bleeding was found in the Sylvian fissure (indicated by bipolar coagulator from left). was suckable. Therefore, it was removed by suction (Fig. 2A). However, a piece of hematoma was stuck to the temporoparietal junction. After the removal of this last hematoma, blood pumped out from cortical artery. It assured us that the hematoma's origin was this cortical artery (Fig. 2B). Right upon this observation, we alleged the radiologist that the hematoma is not related to C-line insertion. Craniotomy bone flap was removed and preserved at $-70^{\circ} \mathrm{C}$ freezer for possible brain swelling. Removed hematoma underwent thorough pathological examination for possible hidden causative material. However, we found nothing but a hematoma.

After the operation, her mental status was improved to GCS of E1VeM4 at postoperative 3 weeks. Brain CT revealed a resolution of midline shift and decreased brain swelling.

The patient then underwent tracheostomy and cranioplasty. Three months after the craniotomy, her neurological status was partially recovered up to GCS of E2VtM4. She was then transferred to other hospital for rehabilitation.

\section{DISCUSSION}

Non-traumatic acute SDH are relatively frequent. There underlying causes include cerebral aneurysm, AVM, brain tumor, hematological disorders, and so on ${ }^{3,8}$. Spontaneous acute $\mathrm{SDH}$ of arterial origin is rare. It can be distinguished from other SDH by the following characteristics". First, arterial bleeding is proved at operative finding or some other indirect evidence such as angiography. Second, the case must not be related to either traumatic history or known underlying vascular disease that could bleed itself such as arterial aneurysm, AVM, tumor, and coagulopathy. In addition, it should not be originated from other types of hemorrhage such as subarachnoid hemorrhage or intracerebral hemorrhage.

In this case, there was intraoperative finding of cortical arterial pumping. In CT, the hemorrhage showed as mixed formation with isodense and hyperdense lesion. In T2-weighted MRI, the hemorrhage appeared to be isointense mixed with hyperintensity, indicating that the hematoma was at hyperacute stage. Considering the time of neurologic deterioration, it took only $30 \mathrm{~min}$ from starting headache to coma state, indicating that there was arterial bleeding.

Why arterial bleeding occurred in this case remains unclear. We have reviewed the central line insertion procedure. However, we did not find anything in the procedure at the left subclavian vein that could cause a cortical artery rupture of right cerebral hemisphere. Neither remarkable blood pressure change nor head trauma during the procedure occurred.

Several possible anatomical situations that might lead to 
cortical artery to rupture without traumatic events have been reported, including: (1) fragile arterial twig branching from cortical artery vulnerable to pressure connecting to dura mater, (2) a knuckle of cortical artery protruding through the arachnoid and adherent to the dura mater, (3) adhesions bet- ween a cortical artery and arachnoid or dura mater ${ }^{5)}$. Intra- operative finding that suggesting one of them was not found in this case.

Spontaneous arterial bleeding related to hypertension ${ }^{2,10}$, alcohol abuse ${ }^{1,9)}$ and the use of anticoagulant or antiplatelet agent have been reported. However, this patient did not have any underlying premedical history except thyroid cancer. In addition, her laboratory findings were within normal ranges.

\section{CONCLUSION}

Although arterial origin spontaneous $\mathrm{SDH}$ is rare, it has been reported in several case reports. Like other cases previously reported, the patient in this case report also had sudden neurologic deterioration and showed poor outcome. A cortical artery bleeding was found in operative field. Therefore, acute SDH without trauma history should be considered as an arterial bleeding. Early operation is recommended.

\section{REFERENCES}

1. Akioka N, Fukuda O, Takaba M, Kameda H, Saito T, Endo S: Clinical investigation of acute spontaneous subdural hematoma cases. J Stroke Cerebrovasc Dis 16:109-113, 2007

2. Arai $\mathrm{H}$ : Acute hypertensive subdural hematoma from arterial rupture shortly after the onset of cerebral subcortical hemorrhage: leakage of contrast medium during angiography. Stroke 14:281285, 1983

3. Avis SP: Nontraumatic acute subdural hematoma. A case report and review of the literature. Am J Forensic Med Pathol 14:130134, 1993

4. Byun HS, Patel PP: Spontaneous subdural hematoma of arterial origin: report of two cases. Neurosurgery 5:611-613, 1979

5. Drake CG: Subdural haematoma from arterial rupture. J Neurosurg 18:597-601, 1961

6. Fell DA, Fitzgerald S, Moiel RH, Caram P: Acute subdural hematomas. Review of 144 cases. J Neurosurg 42:37-42, 1975

7. Hori E, Ogiichi T, Hayashi N, Kuwayama N, Endo S: Case report: acute subdural hematoma due to angiographically unvisualized ruptured aneurysm. Surg Neurol 64:144-146, 2005

8. Kim JH, Gwak HS, Hong EK, Bang CW, Lee SH, Yoo H: A case of benign meningioma presented with subdural hemorrhage. Brain Tumor Res Treat 3:30-33, 2015

9. Koç RK, Paşaoğlu A, Kurtsoy A, Oktem IS, Kavuncu I: Acute spontaneous subdural hematoma of arterial origin: a report of five cases. Surg Neurol 47:9-11, 1997

10. Matsuyama T, Shimomura T, Okumura Y, Sakaki T: Acute subdural hematomas due to rupture of cortical arteries: a study of the points of rupture in 19 cases. Surg Neurol 47:423-427, 1997 